TRANSACTIONS OF THE

AMERICAN MATHEMATICAL SOCIETY

Volume 358, Number 5 , Pages 1921-1935

S 0002-9947(05)04129-2

Article electronically published on December 20, 2005

\title{
ON TOPOLOGICAL INVARIANTS OF STRATIFIED MAPS WITH NON-WITT TARGET
}

\author{
MARKUS BANAGL
}

\begin{abstract}
The Cappell-Shaneson decomposition theorem for self-dual sheaves asserts that on a space with only even-codimensional strata any selfdual sheaf is cobordant to an orthogonal sum of twisted intersection chain sheaves associated to the various strata. In sharp contrast to this result, we prove that on a space with only odd-codimensional strata (not necessarily Witt), any self-dual sheaf is cobordant to an intersection chain sheaf associated to the top stratum: the strata of odd codimension do not contribute terms. As a consequence, we obtain formulae for the pushforward of characteristic classes under a stratified map whose target need not satisfy the Witt space condition. To prove these results, we introduce a new category of superperverse sheaves, which we show to be abelian. Finally, we apply the results to the study of desingularization of non-Witt spaces and exhibit a singular space which admits a PL resolution in the sense of M. Kato, but no resolution by a stratified map.
\end{abstract}

\section{INTRODUCTION}

Generalized Poincaré duality and topological invariants such as L-classes for singular spaces can be obtained from Verdier self-dual complexes of sheaves. Let $X$ be a closed, oriented, Whitney stratified pseudomanifold. Every self-dual complex of sheaves $\mathbf{S}^{\bullet}$ on $X$ has homology L-classes $L_{i}\left(\mathbf{S}^{\bullet}\right) \in H_{i}(X ; \mathbb{Q})$ by the ThomPontrjagin construction; see CS91 or CSW91. If $X$ is a Witt space (that is, the middle-dimensional middle-perversity intersection homology of the links of oddcodimensional strata vanishes), then the middle-perversity intersection chain sheaf $\mathbf{S}^{\bullet}=\mathbf{I} \mathbf{C}_{\bar{m}}^{\bullet}(X)$ is self-dual and $L(X)=L\left(\mathbf{I C}_{\bar{m}}^{\bullet}(X)\right)$ is the Goresky-MacPherson L-class of $X$. If $X$ is not Witt, then $\mathbf{I C}_{\bar{m}}^{\bullet}(X)$ is not self-dual, and it is shown in Ban02 that intersection homology-type invariants on non-Witt spaces are given by a category of sheaves $S D(X)$ whose objects are self-dual and interpolate between $\mathbf{I C}_{\bar{m}}^{\bullet}(X)$ and $\mathbf{I C}_{\bar{n}}^{\bullet}(X)$, where $\bar{m}, \bar{n}$ denote lower- and upper-middle perversity, respectively. This category may or may not be empty, and it can be understood in terms of Lagrangian structures along the strata of odd codimension. Assume $X$ is such that $S D(X) \neq \varnothing$. We proved in Ban05 that, given a normalization on the top stratum, the classes $L_{i}\left(\mathbf{S}^{\bullet}\right)$ are independent of the choice of $\mathbf{S}^{\bullet} \in S D(X)$. Consequently, a non-Witt space has a well-defined L-class $L(X)$, provided $S D(X) \neq \varnothing$.

Received by the editors February 11, 2003.

2000 Mathematics Subject Classification. Primary 57R20, 55N33.

Key words and phrases. Stratified maps, signature, characteristic classes, intersection homology, self-dual sheaves, perverse sheaves, t-structures, desingularization, cobordism of sheaves.

The author was supported in part by NSF Grant DMS-0072550. 
Among the results of the present paper is the following:

Theorem 1.1. Let $X^{n}$ and $Y^{m}$ be closed, oriented, Whitney stratified pseudomanifolds such that $m-n$ is even and $S D(Y) \neq \varnothing$ (for instance $Y$ a Witt space). If $f: Y \rightarrow X$ is a stratified map and $X$ has only strata of odd codimension (except for the top stratum), then

$$
f_{*} L_{i}(Y)=L_{i}(X ; \mathcal{S}),
$$

where $\mathcal{S}$ is a local coefficient system on the top stratum of $X$.

This formula is in striking contrast to the situation where $X$ has only strata of even codimension. Under that assumption, Cappell and Shaneson CS91 show that

$$
f_{*} L_{i}(Y)=\sum_{Z \in \mathcal{X}} L_{i}\left(\bar{Z} ; \mathcal{S}^{Z}\right),
$$

where $\mathcal{X}$ is the set of components of pure strata of $X, \bar{Z}$ is the closure of $Z$ in $X, \mathcal{S}^{Z}$ is a local system on $Z$, and $L_{i}\left(\bar{Z} ; \mathcal{S}^{Z}\right)$ is viewed as a homology class in $X$ via the inclusion $\bar{Z} \hookrightarrow X$. Thus each singular stratum $Z$ of even codimension may contribute a term $L_{i}\left(\bar{Z} ; \mathcal{S}^{Z}\right)$, not just the top stratum. Our Theorem 1.1 introduces the new phenomenon that strata of odd codimension never contribute a term to the pushforward $f_{*} L_{i}(Y)$.

Formulae such as (11) and (2) are readily implied by orthogonal decompositions on the sheaf level. It is in fact sufficient to obtain such decompositions up to algebraic cobordism of self-dual sheaves, since this relation preserves invariants such as the Lclasses. Our methods apply to spaces $X$ that admit a parity-separation, i.e. a cover $X={ }^{o} X \cup{ }^{e} X$ by open sets ${ }^{o} X,{ }^{e} X$, such that ${ }^{o} X$ with the induced stratification has only strata of odd codimension (except for the top stratum), and ${ }^{e} X$ with the induced stratification has only strata of even codimension. Let $\Omega(X)$ be the abelian group of algebraic cobordism classes of self-dual sheaves on $X$. The cobordism class of a sheaf $\mathbf{S}^{\bullet}$ will be denoted by $\left[\mathbf{S}^{\bullet}\right]$. Our decomposition theorem is:

Theorem 1.2. Let $X^{n}$ be a stratified pseudomanifold with parity-separation $X=$ ${ }^{o} X \cup{ }^{e} X$ and let $\boldsymbol{S}^{\bullet}$ be a self-dual sheaf. Then

$$
\left[\boldsymbol{S}^{\bullet}\right]=\left[\boldsymbol{P}^{\bullet}\right] \in \Omega(X)
$$

with

$$
\left.\boldsymbol{P}^{\bullet}\right|_{{ } X} \cong \boldsymbol{I} \boldsymbol{C}_{\mathcal{L}}\left({ }^{o} X ;{ }^{o} \mathcal{S}\right) \in S D\left({ }^{o} X\right)
$$

and

$$
\left.\boldsymbol{P}^{\bullet}\right|_{e X} \cong \bigoplus_{Z \in \in^{e}} \boldsymbol{I} \boldsymbol{C}_{\bar{m}}^{\bullet}\left(\bar{Z} ; \mathcal{S}^{Z}\right)\left[\frac{1}{2} \operatorname{codim} Z\right] .
$$

Here, ${ }^{o} \mathcal{S}$ is a local coefficient system on the top stratum of ${ }^{\circ} X, \boldsymbol{I C}_{\mathcal{L}}{ }^{\circ}$ is a self-dual intersection chain sheaf on ${ }^{\circ} X$ determined by a Lagrangian structure $\mathcal{L}$, and ${ }^{e} \mathcal{X}$ is the set of components of pure strata of ${ }^{e} X$.

If ${ }^{o} X=\varnothing$, then this is the Cappell-Shaneson decomposition. Theorem 1.1 follows from Theorem 1.2 by taking ${ }^{e} X=\varnothing$.

To prove Theorem [1.2, we introduce a new category $S P(X)$ of superperverse sheaves on $X$. This concept can be defined for any pseudomanifold, and we prove 
that $S P(X)$ is an abelian category (Theorem 3.2). For a space with only evencodimensional strata, this category equals the standard category of (middle-) perverse sheaves. We show that $S P(X)$ is big enough so that every self-dual sheaf on $X$ is cobordant to a superperverse one, yet small enough so that on a space with only odd-codimensional strata, every self-dual superperverse sheaf is given by Lagrangian structures.

The decomposition Theorem 1.2 has implications for the desingularization of spaces: It identifies the existence of Lagrangian structures along the strata of odd codimension of $X$ as an obstruction to resolving $X$ by a stratified map $f: M \rightarrow X$, $M$ a manifold.

Theorem 1.3. If $X^{n}$ has a parity-separation and there exists no Lagrangian structure (for constant coefficients) at the strata of odd codimension, then $X$ is not resolvable by a stratified map.

We give a brief review of M. Kato's theory Kat73 of topological (PL-) resolutions and compare Theorem 1.3 to this theory. In particular, we exhibit an example of a singular space $X^{4}$ which admits a PL resolution in the sense of Kato, but no resolution by a stratified map. The author would like to acknowledge a discussion in which he reported some of his initial ideas and evidence for the results presented here to Sylvain Cappell.

\section{A Remark on PERVERSE SheAVES AND Verdier DUALity}

Let $\mathbb{k}$ be a field of characteristic not 2. Let $X$ be a stratified space and $\mathcal{X}$ the set of connected components of pure strata of $X$. The bounded, constructible, derived category of complexes of sheaves of $\mathbb{k}$-vector spaces on $X$ will be denoted by $D(X)$. It possesses an important involution $\mathcal{D}$, the Verdier dualizing functor. The $i$-th cohomology sheaf of a complex of sheaves $\mathbf{A}^{\bullet}$ will be written as $\mathbf{H}^{i}\left(\mathbf{A}^{\bullet}\right)$. Given any function $\bar{p}: \mathcal{X} \rightarrow \mathbb{Z},\left({ }^{\bar{p}} D^{\leq 0}(X),{ }^{\bar{p}} D^{\geq 0}(X)\right)$ is the $\bar{p}$-perverse t-structure on $X$, $\bar{p}_{\tau} \leq 0: D(X) \rightarrow{ }^{\bar{p}} D^{\leq 0}(X)$ and ${ }^{\bar{p}} \tau \geq 0: D(X) \rightarrow{ }^{\bar{p}} D^{\geq 0}(X)$ are the corresponding t-structure truncations, and ${ }^{\bar{p}} P(X)$ is the heart (the abelian category of $\bar{p}$-perverse sheaves on $X)$. The dual perversity $\bar{q}$ is

$$
\bar{q}(S)=-\bar{p}(S)-\operatorname{dim}(S), S \in \mathcal{X} .
$$

It is pointed out in BBD82 that $\mathcal{D}$ restricts as

$$
\mathcal{D}:{ }^{\bar{p}} D^{\leq 0}(X) \longrightarrow{ }^{\bar{q}} D^{\geq 0}(X), \mathcal{D}:{ }^{\bar{p}} D^{\geq 0}(X) \longrightarrow{ }^{\bar{q}} D^{\leq 0}(X),
$$

and there is an isomorphism of functors

$$
\mathcal{D}^{\bar{p}} H^{0} \cong{ }^{\bar{q}} H^{0} \mathcal{D}
$$

with ${ }^{\bar{p}} H^{0}: D(X) \rightarrow{ }^{\bar{p}} P(X)$ denoting $\bar{p}$-perverse cohomology. It is important for our purposes to sharpen these statements somewhat to yield the following formulae (not explicitly stated in [BBD82]):

Lemma 2.1. In $D(X)$, there are isomorphisms

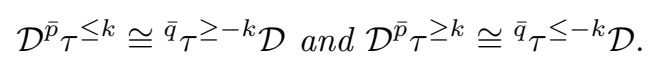

Proof. We will focus on the first of the two isomorphisms. Let $\mathbf{S}^{\bullet} \in D(X)$. It follows from the definition

$$
{ }^{\bar{p}} D^{\leq k}(X)=\left\{\mathbf{X}^{\bullet}: \mathbf{H}^{n+k}\left(\left.\mathbf{X}^{\bullet}\right|_{S}\right)=0, n>\bar{p}(S), \text { all } S \in \mathcal{X}\right\}
$$


and the boundedness of $\mathbf{S}^{\bullet}$ that there exists an integer $N$ such that $\mathbf{S}^{\bullet} \in \bar{p}^{\bar{p}} \leq N(X)$. Consequently, $\mathbf{S}^{\bullet} \cong{ }^{\bar{p}} \tau={ }^{\leq N} \mathbf{S}^{\bullet}, \mathcal{D} \mathbf{S}^{\bullet} \in{ }^{\bar{q}} D^{\geq-N}(X)$ and $\mathcal{D} \mathbf{S}^{\bullet} \cong \bar{q}_{\tau}{ }^{\geq-N} \mathcal{D} \mathbf{S}^{\bullet}$. Thus the claim holds for $k \geq N$. Assume inductively that $\mathcal{D}^{\bar{p}} \tau^{\leq k} \mathbf{S}^{\bullet} \cong \bar{q}_{\tau} \geq-k \mathcal{D} \mathbf{S}^{\bullet}$ has been established and consider the distinguished triangle

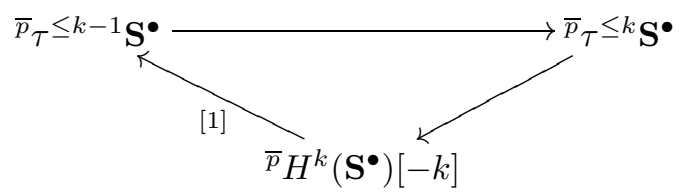

Its dual is

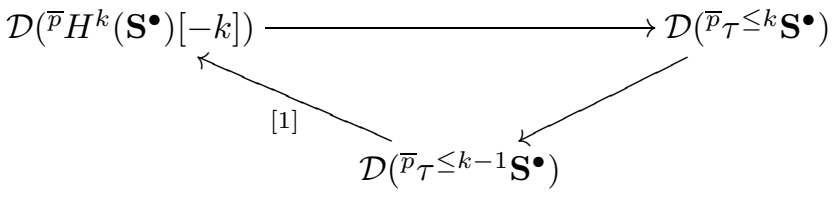

Comparing this triangle to

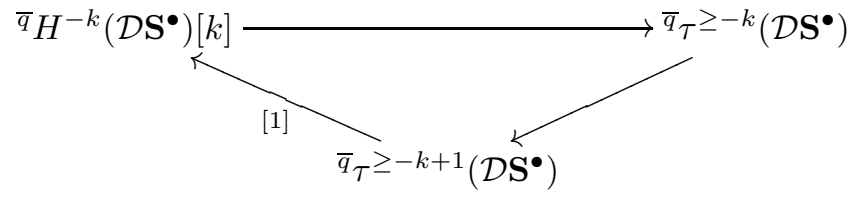

using the induction hypothesis and BBD82

$$
\mathcal{D}\left({ }^{\bar{p}} H^{k}\left(\mathbf{S}^{\bullet}\right)[-k]\right) \cong{ }^{\bar{q}} H^{-k}\left(\mathcal{D} \mathbf{S}^{\bullet}\right)[k],
$$

induces an isomorphism

$$
\mathcal{D}\left(\bar{p}_{\tau} \leq k-1 \mathbf{S}^{\bullet}\right) \cong \bar{q}_{\tau^{\geq-(k-1)}}\left(\mathcal{D} \mathbf{S}^{\bullet}\right)
$$

\section{The CATEGORY OF SUPERPERVERSE SHEAVES}

We will define a category $S P(X)$ of superperverse sheaves on $X$ (possibly not Witt) which is big enough so that every self-dual sheaf on $X$ is cobordant to a superperverse sheaf, yet small enough so that on the class of non-Witt spaces to be considered in this paper (see section 4), every self-dual superperverse sheaf is given by Lagrangian structures in the sense of Ban02.

Let $X$ be a stratified, oriented, topological pseudomanifold without boundary. As all of our sheaves are sheaves of $\mathbb{k}$-vector spaces, the intersection chain sheaves in particular are understood to have $\mathbb{k}$ coefficients. We simply write $\mathbf{I C}_{\bar{m}}^{\bullet}(X)$ instead of $\mathbf{I C}_{\bar{m}}^{\bullet}(X ; \mathbb{k})$. Similarly, the Witt condition is understood with respect to $\mathbb{k}$ coefficients. Thus we simply speak of a "Witt space" instead of a "k-Witt space". If $X$ has only strata of even codimension, then $\mathbf{I C}_{\bar{m}}^{\bullet}(X)$, the intersection chain sheaf with respect to the lower middle perversity $\bar{m}$, is Verdier self-dual, since $\mathbf{I C}_{\bar{m}}^{\bullet}(X)=\mathbf{I} \mathbf{C}_{\bar{n}}^{\bullet}(X)$, the intersection chain sheaf with respect to the upper middle perversity $\bar{n}$. More generally, $\mathbf{I C}_{\bar{m}}^{\bullet}(X)$ is still self-dual on $X$ if $X$ is a Witt space. If $X$ is not a Witt space, then the canonical morphism $\mathbf{I} \mathbf{C}_{\bar{m}}^{\bullet}(X) \rightarrow \mathbf{I} \mathbf{C}_{\bar{n}}^{\bullet}(X)$ is not an isomorphism and $\mathbf{I C}_{\bar{m}}^{\bullet}(X)$ is not self-dual. 
We will briefly review some relevant results of Ban02, where a theory of intersection homology-type invariants for non-Witt spaces is developed. Let $X^{n}$ be an $n$-dimensional pseudomanifold with a fixed stratification

$$
X=X_{n} \supset X_{n-2} \supset X_{n-3} \supset \ldots \supset X_{0} \supset X_{-1}=\varnothing
$$

such that $X_{j}$ is closed in $X$ and $X_{j}-X_{j-1}$ is an open manifold of dimension $j$. Set $U_{k}=X-X_{n-k}$. Throughout this paper we will follow the indexing conventions of [GM83]. Thus self-duality will be understood with respect to $\mathcal{D}[n]$, rather than with respect to $\mathcal{D}$.

As shown in [Ban02, intersection homology-type invariants on non-Witt spaces are given by objects of a certain full subcategory $S D(X) \subset D(X)$. The objects of this category satisfy two properties: On the one hand, they are self-dual, on the other hand, they are as close to the middle perversity intersection chain sheaves as possible, that is, interpolate between $\mathbf{I C}_{\bar{m}}^{\bullet}(X)$ and $\mathbf{I C}_{\bar{n}}^{\bullet}(X)$. The precise definition is as follows:

Definition 3.1. Let $S D(X)$ be the full subcategory of $D(X)$ whose objects $\mathbf{S}^{\bullet}$ satisfy the following axioms:

(SD1): Top stratum: $\left.\left.\mathbf{S}^{\bullet}\right|_{U_{2}} \cong \mathbf{H}^{-n}\left(\mathbf{S}^{\bullet}\right)[n]\right|_{U_{2}}$.

(SD2): Lower bound: $\mathbf{H}^{i}\left(\mathbf{S}^{\bullet}\right)=0$, for $i<-n$.

(SD3): Stalk condition for the upper middle perversity $\bar{n}$ :

$\mathbf{H}^{i}\left(\left.\mathbf{S}^{\bullet}\right|_{U_{k+1}}\right)=0$, for $i>\bar{n}(k)-n, k \geq 2$.

(SD4): Self-duality: $\mathbf{S}^{\bullet}$ has an associated isomorphism

$$
d: \mathcal{D} \mathbf{S}^{\bullet}[n] \stackrel{\cong}{\longrightarrow} \mathbf{S}^{\bullet} \text { such that } \mathcal{D} d[n]= \pm d .
$$

Depending on $X$, the category $S D(X)$ may or may not be empty. If $\mathbf{S}^{\bullet} \in S D(X)$, then there exist morphisms $\mathbf{I C}_{\bar{m}}^{\bullet}(X ; \mathcal{S}) \stackrel{\alpha}{\longrightarrow} \mathbf{S}^{\bullet} \stackrel{\beta}{\longrightarrow} \mathbf{I C}_{\bar{n}}^{\bullet}(X ; \mathcal{S})$, where $\mathcal{S}$ is the selfdual local coefficient system $\mathcal{S}=\left.\mathbf{H}^{-n}\left(\mathbf{S}^{\bullet}\right)\right|_{U_{2}}$, such that

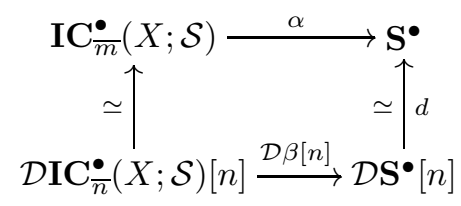

(where $d$ is given by (SD4)) commutes, which clarifies the relation between intersection chain sheaves and objects of $S D(X)$. The main structure theorem on $S D(X)$ is a description as a Postnikov system with fibers given by categories of Lagrangian structures along the strata of odd codimension (for more details we refer the reader to Ban02]):

Theorem 3.1. Let $n=\operatorname{dim} X$ be even. There is an equivalence of categories

$S D(X) \simeq \operatorname{Lag}\left(U_{n}-U_{n-1}\right) \rtimes \operatorname{Lag}\left(U_{n-2}-U_{n-3}\right) \rtimes \ldots \rtimes \operatorname{Lag}\left(U_{4}-U_{3}\right) \rtimes \operatorname{Coeff}\left(U_{2}\right)$.

(Similarly for $n$ odd.)

Given a local coefficient system $\mathcal{S} \in \operatorname{Coeff}\left(U_{2}\right)$, we will write $S D(X ; \mathcal{S}) \subset S D(X)$ for the full subcategory of objects $\mathbf{S}^{\bullet}$ such that $\left.\mathbf{S}^{\bullet}\right|_{U_{2}} \cong \mathcal{S}[n]$. In the context of $L^{2}$ cohomology on Riemannian pseudomanifolds, the idea of using Lagrangian subspaces to restore Poincaré duality appears in [Che80]. 
Example 3.1. Let $X^{6}$ be the product of a circle with the (unreduced) suspension of complex projective space, $X^{6}=S^{1} \times \Sigma \mathbb{C} P^{2}$. This space has a stratum of odd codimension 5 consisting of the disjoint union of two circles. The link of this stratum is $\mathbb{C} P^{2}$, and there is no Lagrangian subspace in the middle cohomology $H^{2}\left(\mathbb{C} P^{2} ; \mathbb{k}\right)$. Then the structure theorem implies $S D\left(X^{6}\right)=\varnothing$, so that there is no meaningful way to define intersection homology-type invariants on $X^{6}$.

Now let $X^{6}$ be the product of a circle with the suspension of the product of two 2-spheres, $X^{6}=S^{1} \times \Sigma\left(S^{2} \times S^{2}\right)$. The stratum of odd codimension 5 consists again of the disjoint union of two circles, but with link $S^{2} \times S^{2}$. The form on $H^{2}\left(S^{2} \times S^{2} ; \mathbb{k}\right)$ is a hyperbolic plane, which is split. The structure theorem implies $S D\left(X^{6}\right) \neq \varnothing$. In fact, the functors implicit in the theorem construct a self-dual sheaf on $X^{6}$ for every choice of Lagrangian subspace. For general $\mathbb{k}$, we do not have a signature that tells us that a Lagrangian subspace exists. If char $\mathbb{k}=0$, then one has a chance to order the field. In fact, by the theorem of Artin-Schreier, $\mathbb{k}$ has an ordering iff -1 is not a sum of squares in $\mathbb{k}$. Let $P$ be an ordering on $\mathbb{k}$ and let $V$ be a symmetric inner product space. By the inertia theorem, $V \cong V^{+} \oplus V^{-}$with $V^{+}$positive definite and $V^{-}$negative definite. Then the signature $\sigma_{P}(V)$ of $V$ at the ordering $P$ is defined as $\operatorname{rk} V^{+}-\mathrm{rk} V^{-}$. If $V$ is split, then $\sigma_{P}(V)=0$ for every ordering $P$. Suppose that $P$ is such that every positive element is a square. Then $\sigma_{P}: \operatorname{Witt}(\mathbb{k}) \rightarrow \mathbb{Z}$ is an isomorphism, where $\operatorname{Witt}(\mathbb{k})$ denotes the Witt ring of $\mathbb{k}$. For example, $\sigma: W i t t(\mathbb{R}) \cong \mathbb{Z}$.

It is natural to ask the following question: What is the set of perversities $\bar{r}$ such that every object of $S D(X)$ is $\bar{r}$-perverse? In other words, for which $\bar{r}$ is $S D(X) \subset \bar{r} P(X)$ ? For $S \in \mathcal{X}$ with $k=\operatorname{codim} S$ even, the stalk condition (SD3) implies $\bar{r}(S) \geq \bar{n}(k)-n$ and the costalk condition (using self-duality) implies $\bar{r}(S) \leq$ $\bar{n}(k)-n+2$. Thus $\bar{r}(S)$ may have three values,

$$
\bar{r}(S) \in\{\bar{n}(k)-n, \bar{n}(k)-n+1, \bar{n}(k)-n+2\} .
$$

When one speaks of the category of perverse sheaves on a space with only evencodimensional strata, then one refers to ${ }^{\bar{r}} P(X)$ with the middle value

$$
\bar{r}(S)=\bar{n}(k)-n+1=\frac{1}{2} \operatorname{codim} S-\operatorname{dim} X,
$$

since this value yields a self-dual perversity. The situation for $S \in \mathcal{X}$ with $k=$ codim $S$ odd is quite different. The stalk condition gives the same lower bound as before, but the costalk condition produces $\bar{r}(S) \leq \bar{n}(k)-n+1$ as an upper bound, so that $\bar{r}(S)$ may have precisely the two values

$$
\bar{r}(S) \in\{\bar{n}(k)-n, \bar{n}(k)-n+1\} .
$$

Definition 3.2. Throughout the remainder of this article, $\bar{p}, \bar{q}: \mathcal{X} \rightarrow \mathbb{Z}$ will denote the perversity functions given by

$$
\begin{aligned}
& \bar{p}(S)= \begin{cases}\bar{n}(\operatorname{codim} S)-n+1, & \text { codim } S \text { even }, \\
\bar{n}(\operatorname{codim} S)-n, & \operatorname{codim} S \text { odd },\end{cases} \\
& \bar{q}(S)=\bar{n}(\operatorname{codim} S)-n+1,
\end{aligned}
$$

if $S \in \mathcal{X}$ is not contained in the top stratum, and $\bar{p}(S)=\bar{q}(S)=-n$ for $S$ a component of the top stratum. 
We note that $\bar{p}$ and $\bar{q}$ are dual, since for $S \in \mathcal{X}, k=\operatorname{codim} S$ odd, the dual value is

$$
\begin{aligned}
\bar{p}^{*}(S) & =-\bar{p}(S)-\operatorname{dim} S-\operatorname{dim} X \\
& =-\left(\frac{1}{2}(k-1)-n\right)-(n-k)-n \\
& =\bar{n}(k)-n+1 \\
& =\bar{q}(S) .
\end{aligned}
$$

By construction, we have

$$
S D(X) \subset{ }^{\bar{p}} P(X) \cap{ }^{\bar{q}} P(X) .
$$

Definition 3.3. The category of superperverse sheaves on $X$ is the full subcategory

$$
S P(X)={ }^{\bar{p}} D^{\leq 0}(X) \cap{ }^{\bar{q}} D^{\geq 0}(X) \subset D(X) .
$$

If $X$ is a space with only even-codimensional strata, for example a complex algebraic variety, then $\bar{p}=\bar{q}$ and $S P(X)={ }^{\bar{p}} P(X)={ }^{\bar{q}} P(X)$ is the usual category of perverse sheaves.

Theorem 3.2. $S P(X)$ is an abelian category.

Proof. There is an obvious inclusion ${ }^{\bar{p}} P(X) \cap{ }^{\bar{q}} P(X) \subset S P(X)$. The converse inclusion is implied by ${ }^{\bar{q}} D^{\geq 0} \subset{ }^{\bar{p}} D^{\geq 0}$ and ${ }^{\bar{p}} D^{\leq 0} \subset{ }^{\bar{q}} D^{\leq 0}$. Thus we can represent superperverse sheaves as

$$
S P(X)={ }^{\bar{p}} P(X) \cap{ }^{\bar{q}} P(X) .
$$

Dually to Definition 3.3, we set $S P^{*}(X)={ }^{\bar{p}} D^{\geq 0}(X) \cap \cap^{\bar{q}} D^{\leq 0}(X)$. Verdier duality defines an equivalence of categories $\mathcal{D}[n]: S P(X) \simeq S P^{*}(X)$. The obvious inclusion ${ }^{\bar{p}} P(X) \cap{ }^{\bar{q}} P(X) \subset S P^{*}(X)$ is not strict, as we have $\mathcal{D}[n]: S P^{*}(X) \rightarrow{ }^{\bar{p}} P(X) \cap$ ${ }^{\bar{q}} P(X)$ and $\mathcal{D}[n]$ preserves the subcategory ${ }^{\bar{p}} P(X) \cap{ }^{\bar{q}} P(X)$. Hence

$$
S P(X)=S P^{*}(X) \text {. }
$$

The category $S P$ is additive, since ${ }^{\bar{p}} P$ and ${ }^{\bar{q}} P$ are additive. Let $f: \mathbf{X}^{\bullet} \rightarrow \mathbf{Y}^{\bullet}$ be a morphism in $S P$. We will show that it possesses a cokernel and a kernel in $S P$. Embedding $f$ in a distinguished triangle

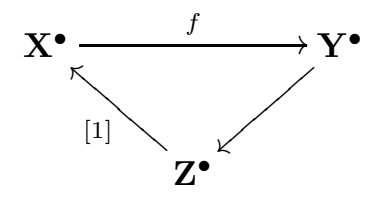

we have

$$
\mathbf{Z}^{\bullet} \in{ }^{\bar{p}} D^{\leq 0} \cap{ }^{\bar{p}} D^{\geq-1} \cap{ }^{\bar{q}} D^{\leq 0} \cap{ }^{\bar{q}} D^{\geq-1} .
$$

The sheaf ${ }^{\bar{q}} H^{0}\left(\mathbf{Z}^{\bullet}\right) \cong{ }^{\bar{q}} \tau={ }^{\geq 0} \mathbf{Z}^{\bullet}$ is a cokernel for $f$ in ${ }^{\bar{q}} P$, but $\mathbf{Z}^{\bullet} \cong{ }^{\bar{p}} \tau=0$ plies that it is an object in $S P$ and hence is a cokernel for $f$ in $S P$. Similarly, ${ }^{\bar{q}} H^{0}\left(\mathbf{Z}^{\bullet}[-1]\right) \cong{ }^{\bar{q}} \tau \leq 0\left(\mathbf{Z}^{\bullet}[-1]\right)$ is a kernel for $f$ in ${ }^{\bar{q}} P$, but $\mathbf{Z}^{\bullet}[-1] \cong \bar{p}_{\tau} \geq 0\left(\mathbf{Z}^{\bullet}[-1]\right)$ implies that it is an object in $S P^{*}=S P$ and hence is a kernel for $f$ in $S P$. Using fullness of $S P$ in ${ }^{\bar{q}} P$, the isomorphism $\operatorname{coim} f \cong \operatorname{im} f$ in the abelian category ${ }^{\bar{q}} P(X)$ is a morphism in $S P$. 


\section{Parity-Separated SPACES}

The chief results of the present paper will apply to the following class of spaces:

Definition 4.1. A parity-separation on a stratified space $X$ is a decomposition

$$
X={ }^{o} X \cup{ }^{e} X
$$

into open subsets ${ }^{o} X,{ }^{e} X \subset X$ such that ${ }^{o} X$ with the induced stratification has only strata of odd codimension (except for the top stratum), and ${ }^{e} X$ with the induced stratification has only strata of even codimension.

Alternatively, $X$ has a parity-separation iff the closure $\bar{S}$ of any stratum $S \in \mathcal{X}$, different from the top stratum, contains only strata of its own kind with respect to the parity of codimension. If $X={ }^{o} X \cup{ }^{e} X$ is a parity-separation, then ${ }^{o} \mathcal{X}$ will denote the set of components of pure strata of ${ }^{o} X$ and ${ }^{e} \mathcal{X}$ will denote the set of components of pure strata of ${ }^{e} X$.

Example 4.1. Let $K$ be a real quadratic number field and $\mathcal{O}_{K}$ the ring of algebraic integers in $K$. The Hilbert modular group $\Gamma=P S L_{2}\left(\mathcal{O}_{K}\right)$ acts on the product $H \times H$ of two upper half planes by

$$
(z, w) \mapsto\left(\frac{a z+b}{c z+d}, \frac{\bar{a} w+\bar{b}}{\bar{c} w+\bar{d}}\right),
$$

where - denotes the non-trivial element of the Galois group $\operatorname{Gal}(K / \mathbb{Q})$. The Hilbert modular surface of $K$ is the orbit space $X^{4}=(H \times H) / \Gamma$. It is non-compact and has finitely many singular points, the cusps. Various compactifications have been studied: The reductive Borel-Serre compactification $\bar{X}$ has the advantage that the Hecke operators extend to this compactification. In constructing $\bar{X}$, one adjoins certain boundary circles to $X$. One obtains a stratification $\bar{X}_{4} \supset \bar{X}_{1} \supset \bar{X}_{0} \supset \varnothing$ by taking $\bar{X}_{1}-\bar{X}_{0}$ to be the disjoint union of the boundary circles and $\bar{X}_{0}$ to be the cusps. The links of the components of $\bar{X}_{1}-\bar{X}_{0}$ are tori; hence $\bar{X}^{4}$ is not a Witt space. However, it possesses a parity-separation: Take ${ }^{o} \bar{X}=\bar{X}_{4}-\bar{X}_{0}$ and take $e^{\bar{X}}$ to be the union of small open neighborhoods of the cusps in $X$. It is shown in BK04 that $S D(\bar{X} ; \mathbb{R}) \neq \varnothing$.

Let $D^{\mathcal{D}}(X)$ denote the full subcategory of $D(X)$ containing all objects invariant under the action of $\mathcal{D}[n]$, i.e. $D^{\mathcal{D}}(X)$ contains all self-dual sheaves on $X$ (without any stalk restrictions).

Proposition 4.1. If $X$ possesses a parity-separation, then

$$
D^{\mathcal{D}}\left({ }^{o} X\right) \cap S P\left({ }^{o} X\right)=S D\left({ }^{o} X\right),
$$

i.e. any self-dual superperverse sheaf on a space with only odd-codimensional strata (excluding the top stratum) is isomorphic to a sheaf $\boldsymbol{I C}_{\mathcal{L}}^{\bullet}(X ; \mathcal{S})$ determined by Lagrangian structures $\mathcal{L}$ (see Theorem 3.1) and given on the top stratum by a local coefficient system $\mathcal{S}$.

Proof. Any object of $S D\left({ }^{o} X\right)$ is in $S P\left({ }^{o} X\right)$ by (3) in section 3 and using $S P=$ ${ }^{\bar{p}} P \cap{ }^{\bar{q}} P$ (established in the proof of Theorem 3.2$)$. Any object of $S D\left({ }^{\circ} X\right)$ is in $D^{\mathcal{D}}\left({ }^{o} X\right)$ by (SD4). Let $\mathbf{S}^{\bullet} \in D^{\mathcal{D}}\left({ }^{o} X\right) \cap S P\left({ }^{o} X\right)$. Since $\mathbf{S}^{\bullet} \in \bar{p} D^{\leq 0}\left({ }^{o} X\right)$, we have $\mathbf{H}^{i}\left(\left.\mathbf{S}^{\bullet}\right|_{U_{2} \cap^{o} X}\right)=0$ for $i>\bar{p}\left(U_{2} \cap{ }^{o} X\right)=-n$, and since $\mathbf{S}^{\bullet} \in{ }^{\bar{q}} D^{\geq 0}\left({ }^{o} X\right)$, we have $\mathbf{H}^{i}\left(\left.\mathbf{S}^{\bullet}\right|_{U_{2} \cap^{\circ} X}\right)=0$ for $i<\bar{q}\left(U_{2} \cap^{o} X\right)=-n$, establishing (SD1). We prove the lower bound axiom (SD2) by induction on the codimension $k$ of strata. The induction 
start is furnished by (SD1). Suppose that $\mathbf{H}^{i}\left(\left.\mathbf{S}^{\bullet}\right|_{U_{k}}\right)$ vanishes for $i<-n$. If $j$ denotes the open inclusion $j: U_{k} \hookrightarrow U_{k+1}$ and $i: U_{k+1}-U_{k} \hookrightarrow U_{k+1}$ denotes the inclusion of the complement, then the distinguished triangle

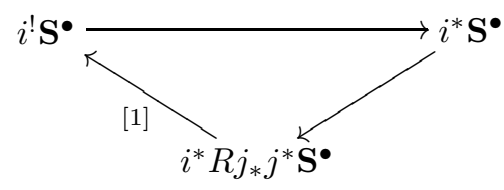

induces an exact sequence

$$
\mathbf{H}^{m}\left(i \mathbf{S}^{\bullet}\right) \longrightarrow \mathbf{H}^{m}\left(i^{*} \mathbf{S}^{\bullet}\right) \longrightarrow \mathbf{H}^{m}\left(i^{*} R j_{*} j^{*} \mathbf{S}^{\bullet}\right) .
$$

If $m<-n$, then $\mathbf{H}^{m}\left(i ! \mathbf{S}^{\bullet}\right)=0$ since $\mathbf{S}^{\bullet} \in{ }^{\bar{q}} D^{\geq 0}$ and the value of $\bar{q}$ on a component of $U_{k+1}-U_{k}$ is larger than $-n$. The term $\mathbf{H}^{m}\left(i^{*} R j_{*} j^{*} \mathbf{S}^{\bullet}\right)$ vanishes when $m<-n$ by the induction hypothesis. Thus $\mathbf{H}^{m}\left(\left.\mathbf{S}^{\bullet}\right|_{U_{k+1}}\right)=0$ for $m<-n$. The stalk condition (SD3) is verified by observing that since ${ }^{o} X$ has only strata of odd codimension, the value of $\bar{p}$ on any component of a stratum of ${ }^{o} X$ is given by $\bar{n}(k)-n$, where $k$ is the codimension (see Definition 3.2). Finally, (SD4) is equivalent to $\mathbf{S}^{\bullet} \in D^{\mathcal{D}}$.

Remark 4.1. The inclusion $S D(X) \subset D^{\mathcal{D}}(X) \cap S P(X)$ holds for any pseudomanifold $X$, but it is generally strict if $X$ has singular strata of even codimension: Let $X^{n}$ be a two-strata space with singular stratum $\Sigma$, a manifold of dimension $n-2 c$. The sheaf

$$
\mathbf{S}^{\bullet}=j_{*} \mathbb{k}_{\Sigma}[n-c],
$$

where $j: \Sigma \hookrightarrow X$ is the closed inclusion, is self-dual on $X$,

$$
\begin{aligned}
\mathcal{D}_{X} \mathbf{S}^{\bullet}[n] & =\mathcal{D}_{X}\left(j_{*} \mathbb{k}_{\Sigma}[n-c]\right)[n] \cong j_{!}\left(\mathcal{D}_{\Sigma} \mathbb{k}_{\Sigma}\right)[c] \\
& \cong j_{*} \mathbb{k}_{\Sigma}[\operatorname{dim} \Sigma][c] \cong j_{*} \mathbb{k}_{\Sigma}[n-2 c][c] \\
& \cong \mathbf{S}^{\bullet}
\end{aligned}
$$

and is superperverse as $\left.\mathbf{S}^{\bullet}\right|_{X-\Sigma}=0$ and $\mathbf{H}^{i}\left(\left.\mathbf{S}^{\bullet}\right|_{\Sigma}\right)=0$ for $i>\bar{p}(\Sigma)=c-n$. However, $\mathbf{S}^{\bullet} \notin S D(X)$ since $\mathbf{H}^{i}\left(\left.\mathbf{S}^{\bullet}\right|_{\Sigma}\right) \cong \mathbb{k}_{\Sigma} \neq 0$ for $i=c-n$, violating axiom (SD3).

\section{An ALgEBRAIC COBORDISM CONSTRUCTION}

Let $\Omega(X)$ be the abelian group of algebraic cobordism classes of self-dual sheaves on $X$, CS91. The cobordism class of $\mathbf{S}^{\bullet} \in D^{\mathcal{D}}(X)$ will be written as $\left[\mathbf{S}^{\bullet}\right.$ ] (thus the self-duality isomorphism will be suppressed in the notation).

Theorem 5.1. Let $X^{n}$ be a topological, stratified pseudomanifold and let $\boldsymbol{S}^{\bullet}$ be any self-dual sheaf in $D(X)$. Then

$$
\left[\boldsymbol{S}^{\bullet}\right]=\left[\boldsymbol{P}^{\bullet}\right] \in \Omega(X)
$$

for some superperverse sheaf $\boldsymbol{P}^{\bullet}$.

Proof. Given $\mathbf{S}^{\bullet} \in D^{\mathcal{D}}(X)$, we embed the canonical morphisms

$$
{ }^{\bar{p}}{ }^{\leq 0} \mathbf{S}^{\bullet} \longrightarrow \mathbf{S}^{\bullet} \longrightarrow{ }^{\bar{q}} \tau^{\geq 0} \mathbf{S}^{\bullet}
$$


in distinguished triangles:

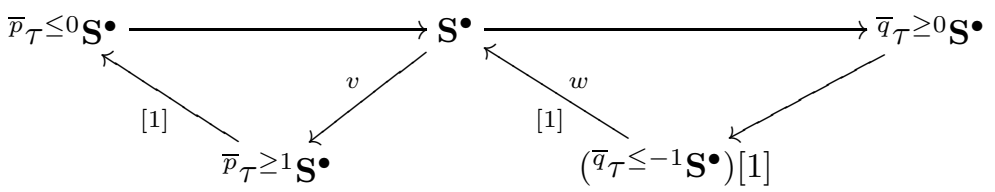

We claim that

$$
{ }_{\bar{q}} \tau^{\leq-1} \mathbf{S}^{\bullet} \stackrel{u}{\longrightarrow} \mathbf{S}^{\bullet} \stackrel{v}{\longrightarrow}{ }^{\bar{p}} \tau^{\geq 1} \mathbf{S}^{\bullet},
$$

where $u=w[-1]$, sets up an elementary cobordism. If $D$ is any triangulated category and $\left(D^{\leq 0}, D^{\geq 0}\right)$ a t-structure on it, then $\operatorname{Hom}_{D}(X, Y)=0$ whenever $X \in D^{\leq 0}$ and $Y \in D^{\geq 1}$. Now ${ }^{\bar{q}} \tau \leq-1 \mathbf{S}^{\bullet} \in{ }^{\bar{q}} D^{\leq-1} \subset{ }^{\bar{p}} D^{\leq 0}, \bar{p}_{\tau} \geq 1 \mathbf{S}^{\bullet} \in{ }^{\bar{p}} D^{\geq 1}$ and so

$$
\operatorname{Hom}_{D(X)}\left({ }^{\bar{q}} \tau^{\leq-1} \mathbf{S}^{\bullet},{ }^{\bar{p}} \tau^{\geq 1} \mathbf{S}^{\bullet}\right)=0,
$$

which implies $v u=0$. The duality $\mathcal{D} v[n]=u$ follows from Lemma 2.1. Thus (4) induces a cobordism from $\mathbf{S}^{\bullet}$ to a self-dual sheaf $\mathbf{C}_{u, v}^{\bullet}$,

$$
\left[\mathbf{S}^{\bullet}\right]=\left[\mathbf{C}_{u, v}^{\bullet}\right] \in \Omega(X) .
$$

The remaining task is to show that $\mathbf{C}_{u, v}^{\bullet}$ is superperverse. Since $v u=0$, there exists a lift $u^{\prime}$ of $u$,

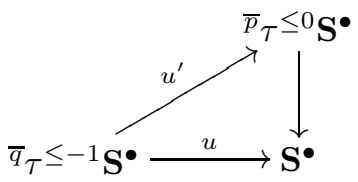

and $\mathbf{C}_{u, v}^{\bullet}$ is isomorphic to the mapping cone $\mathbf{C}_{u^{\prime}}^{\bullet}$ of $u^{\prime}$. The lift is unique as $\operatorname{Hom}\left({ }^{\bar{q}} \tau \leq-1 \mathbf{S}^{\bullet},\left({ }^{\bar{p}} \tau^{21} \mathbf{S}^{\bullet}\right)[-1]\right)=0$. The relation ${ }^{\bar{q}} D^{\leq-1} \subset{ }^{\bar{p}} D^{\leq 0}$ implies an isomorphism of functors

$$
\bar{q}_{\tau} \leq-1{ }^{\bar{p}} \overline{ }^{\leq 0} \cong \bar{q} \tau^{\leq-1} .
$$

Under this identification, we recognize $u^{\prime}$ as the canonical morphism

$$
{ }^{\bar{q}} \tau^{\leq-1}\left(\bar{p}^{\bar{p}} \leq 0 \mathbf{S}^{\bullet}\right) \stackrel{u^{\prime}}{\longrightarrow} \bar{p}_{\tau}^{\leq 0} \mathbf{S}^{\bullet} .
$$

From the distinguished triangle

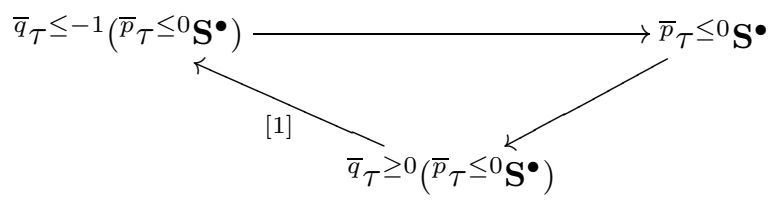

we conclude

$$
\mathbf{C}_{u, v}^{\bullet} \cong \mathbf{C}_{u^{\prime}}^{\bullet} \cong \bar{q}_{\tau} \geq 0 \bar{p} \tau^{\leq 0} \mathbf{S}^{\bullet} .
$$

Clearly, $\mathbf{P}^{\bullet}={ }^{\bar{q}} \tau=0 \bar{p} \tau \leq 0 \mathbf{S}^{\bullet}$ is in ${ }^{\bar{q}} D^{\geq 0}$. Since $\mathbf{P}^{\bullet}$ is self-dual and $\mathcal{D}[n]$ maps ${ }^{\bar{q}} D^{\geq 0} \rightarrow$ ${ }^{\bar{p}} D^{\leq 0}, \mathbf{P}^{\bullet}$ is also in ${ }^{\bar{p}} D^{\leq 0}$ and hence superperverse. 


\section{Characteristic Classes AND Stratified MAPS}

Theorem 6.1. Let $X^{n}$ be a stratified pseudomanifold with parity-separation $X=$ ${ }^{o} X \cup{ }^{e} X$ and $\boldsymbol{S}^{\bullet} \in D(X)$ a self-dual sheaf. Then

$$
\left[\boldsymbol{S}^{\bullet}\right]=\left[\boldsymbol{P}^{\bullet}\right] \in \Omega(X)
$$

with

and

$$
\left.\boldsymbol{P}^{\bullet}\right|_{{ }^{o} X} \cong \boldsymbol{I} C_{\mathcal{L}}\left({ }^{o} X ;{ }^{o} \mathcal{S}\right) \in S D\left({ }^{o} X\right)
$$

$$
\left.\boldsymbol{P}^{\bullet}\right|_{e_{X}} \cong \bigoplus_{Z \in^{e} \mathcal{X}} j_{*} \boldsymbol{I} \boldsymbol{C}_{\bar{m}}^{\bullet}\left(\bar{Z} ; \mathcal{S}^{Z}\right)\left[\frac{1}{2} \operatorname{codim} Z\right]
$$

Here, ${ }^{o} \mathcal{S}$ is a local coefficient system on the top stratum of ${ }^{\circ} X, I \boldsymbol{I C}_{\mathcal{L}}$ is a selfdual intersection chain sheaf on ${ }^{\circ} X$ determined by a Lagrangian structure $\mathcal{L}$ (see Theorem [3.1), $\bar{Z}$ is the closure of $Z, j: \bar{Z} \hookrightarrow{ }^{e} X$ is the inclusion, $\mathcal{S}^{Z}$ is a local coefficient system on $Z$, and $\mathbf{I} \boldsymbol{C}_{\bar{m}}^{\bullet}$ is the middle perversity intersection chain sheaf.

To paraphrase: The result says that only strata of even codimension can contribute terms in the decomposition, while the strata of odd codimension will never contribute any terms other than the term associated to the top stratum. For more information on the decomposition on ${ }^{e} X$, in particular for a description of the local systems, we refer the reader to [CS91] and BCS03.

Proof of Theorem 6.1. By Theorem [5.1, $\left[\mathbf{S}^{\bullet}\right]=\left[\mathbf{T}^{\bullet}\right] \in \Omega(X)$ with $\mathbf{T}^{\bullet} \in S P(X)$. The latter sheaf has restrictions $\mathbf{U}^{\bullet}=\left.\mathbf{T}^{\bullet}\right|_{{ } X} \in S P\left({ }^{o} X\right) \cap D^{\mathcal{D}}\left({ }^{o} X\right)$ and $\mathbf{V}^{\bullet}=$ $\left.\mathbf{T}^{\bullet}\right|_{e_{X}} \in S P\left({ }^{e} X\right) \cap D^{\mathcal{D}}\left({ }^{e} X\right)$. According to Proposition 4.1. $\mathbf{U}^{\bullet} \in S D\left({ }^{o} X\right)$, and by Theorem 3.1

$$
\mathbf{U}^{\bullet} \cong \mathbf{I C}_{\mathcal{L}}^{\bullet}\left({ }^{o} X ;{ }^{o} \mathcal{S}\right)
$$

for some Lagrangian structure $\mathcal{L}$ and local coefficient system ${ }^{\circ} \mathcal{S}$. For the piece containing only strata of even codimension, $S P\left({ }^{e} X\right)={ }^{\bar{q}} P\left({ }^{e} X\right)$, and the decomposition

$$
\begin{gathered}
{\left[\mathbf{V}^{\bullet}\right]=\left[\mathbf{V}_{0}^{\bullet}\right] \in \Omega\left({ }^{e} X\right),} \\
\mathbf{V}_{0}^{\bullet} \cong \bigoplus_{Z \in^{e} \mathcal{X}} j_{*} \mathbf{I C}_{\bar{m}}^{\bullet}\left(\bar{Z} ; \mathcal{S}^{Z}\right)\left[\frac{1}{2} \operatorname{codim} Z\right]
\end{gathered}
$$

is the central result of [CS91]. The overlap ${ }^{\circ} X \cap{ }^{e} X$ is contained in the top stratum, so that

$$
\left.\mathbf{U}^{\bullet}\right|_{{ }^{X} \cap{ }^{e} X} \cong \mathbf{V}_{0}^{\bullet} \mid X \cap^{e} X
$$

Now ${ }^{\bar{q}} P(X)$ is a stack. Therefore there exists a sheaf $\mathbf{P}^{\bullet}$ on $X$ such that $\left.\mathbf{P}^{\bullet}\right|_{{ }^{\prime}} \cong \mathbf{U}^{\bullet}$ and $\left.\mathbf{P}^{\bullet}\right|_{e_{X}} \cong \mathbf{V}_{0}^{\bullet}$.

For the remainder of this paper we assume that every space is Whitney stratified, and that $\mathbb{k}$ is an ordered field. We denote the ordering by $P$. It will remain fixed throughout. This includes all fields where -1 is not a sum of squares, but excludes finite fields and algebraically closed fields. The term stratified map is understood in the sense of Whitney stratification theory. Let $\mathbf{S}^{\bullet} \in D(X)$ be any self-dual sheaf and suppose $\operatorname{dim} X=n=2 k$. The self-duality isomorphism induces a nondegenerate, bilinear pairing on the middle-dimensional hypercohomology $\mathbb{k}$-vector spaces of $\mathbf{S}^{\bullet}$,

$$
\mathcal{H}^{-k}\left(X ; \mathbf{S}^{\bullet}\right) \otimes \mathcal{H}^{-k}\left(X ; \mathbf{S}^{\bullet}\right) \longrightarrow \mathbb{k}
$$

which has a signature $\sigma\left(\mathbf{S}^{\bullet}\right)=\sigma_{P}\left(\mathbf{S}^{\bullet}\right)$, using the ordering $P$ (see the remarks on ordered fields and signatures in Example 3.1). In the following, we will omit 
the ordering from our notation. Using this signature, $\mathbf{S}^{\bullet}$ has homology L-classes $L_{i}\left(\mathbf{S}^{\bullet}\right) \in H_{i}(X ; \mathbb{Q})$, by the Thom-Pontrjagin construction. For example, the intersection chain sheaf $\mathbf{I C}_{\bar{m}}^{\bullet}(X)$ is self-dual on a Witt space, and its L-classes $L_{i}\left(\mathbf{I C}_{\bar{m}}^{\bullet}(X)\right)$ are the Goresky-MacPherson L-classes $L_{i}(X)$ of $X$. An investigation of the L-class of non-Witt spaces has been conducted in Ban05:

Theorem 6.2. Let $X^{n}$ be a closed, oriented pseudomanifold and let $\mathcal{S}$ be a selfdual local system on the top stratum of $X$. If $S D(X ; \mathcal{S}) \neq \varnothing$, then the L-classes $L_{i}(X ; \mathcal{S})=L_{i}\left(\boldsymbol{I} \boldsymbol{C}_{\mathcal{L}}^{\bullet}(X ; \mathcal{S})\right) \in H_{i}(X ; \mathbb{Q}), I_{\mathcal{L}}^{\bullet}(X ; \mathcal{S}) \in S D(X ; \mathcal{S})$, are independent of the choice of Lagrangian structure $\mathcal{L}$.

Thus a non-Witt space has a well-defined L-class $L(X)$, provided $S D(X ; \mathbb{k}) \neq \varnothing$.

Theorem 6.3. Let $X^{n}$ and $Y^{m}$ be closed, oriented pseudomanifolds such that $m-n$ is even and $S D(Y) \neq \varnothing$ (for instance $Y$ a Witt space). If $f: Y \rightarrow X$ is a stratified map and $X$ has only strata of odd codimension (except for the top stratum), then

$$
f_{*} L(Y)=L\left(X ; \mathcal{S}^{X-\Sigma}\right) .
$$

Proof. Let $\mathbf{S}^{\bullet} \in S D(Y)$ be the sheaf on $Y$ such that $L(Y)=L\left(\mathbf{S}^{\bullet}\right)$ (e.g. $\mathbf{S}^{\bullet}=$ $\mathbf{I C}_{\bar{m}}^{\bullet}(Y)$ if $Y$ is Witt). The pushforward $R f_{*} \mathbf{S}^{\bullet}\left[\frac{1}{2}(n-m)\right]$ is again self-dual on $X$, since $f$ is proper. As $X$ has only strata of odd codimension, it admits a parityseparation $X={ }^{o} X \cup{ }^{e} X$ with ${ }^{e} X=\varnothing$. By Theorem [6.1, $R f_{*} \mathbf{S}^{\bullet}\left[\frac{1}{2}(n-m)\right]$ is cobordant to $\mathbf{I C}_{\mathcal{L}}^{\bullet}\left(X ; \mathcal{S}^{X-\Sigma}\right)$ for some Lagrangian structure $\mathcal{L}$. Then $f_{*} L\left(\mathbf{S}^{\bullet}\right)=$ $L\left(R f_{*} \mathbf{S}^{\bullet}\left[\frac{1}{2}(n-m)\right]\right)=L\left(\mathbf{I C}_{\mathcal{L}}^{\bullet}\left(X ; \mathcal{S}^{X-\Sigma}\right)\right)=L\left(X ; \mathcal{S}^{X-\Sigma}\right)$, since cobordism preserves L-classes, and using Theorem 6.2 .

It is again worthwhile to contrast this result with the corresponding statement for spaces with only even-codimensional strata. Let $X^{n}$ be a space with only evencodimensional strata, $\mathcal{X}$ the set of components of pure strata of $X, Y$ a Witt space, and $f: Y \rightarrow X$ a stratified map of even relative dimension. Then the L-classes are related by the formula

$$
f_{*} L_{i}(Y)=\sum_{Z \in \mathcal{X}} L_{i}\left(\bar{Z} ; \mathcal{S}^{Z}\right) ;
$$

see CS91. Theorem 6.3 introduces the new phenomenon that for spaces $X$ with only odd-codimensional strata, no term $L_{i}\left(\bar{Z} ; \mathcal{S}^{Z}\right)$ arises for $Z$ of positive codimension.

Example 6.1. We illustrate Theorem 6.3 with a simple toy-example. For this example, we shall work over $\mathbb{R}$. Let $K 3$ be the Kummer surface,

$$
K 3=\left\{\left[z_{0}: z_{1}: z_{2}: z_{3}\right] \in \mathbb{C} P^{3} \mid z_{0}^{4}+z_{1}^{4}+z_{2}^{4}+z_{3}^{4}=0\right\},
$$

a simply connected, closed 4-manifold with intersection form $E_{8} \oplus E_{8} \oplus 3 H$ (where $H$ is the standard hyperbolic $2 \times 2$-matrix) and signature $\sigma(K 3)=16$. Let $T^{2} \hookrightarrow K 3$ be the following embedding of a 2-torus: Let $\pi: K 3 \rightarrow \mathbb{C} P^{1}$ be an elliptic fibration with general fiber $T^{2}$ and 24 nodal fibers. Then take $T^{2}=\pi^{-1}(p) \hookrightarrow K 3, p \in \mathbb{C} P^{1}$, to be the inclusion of one of the general fibers. If $\pi_{1}: T^{2} \rightarrow S^{1}$ is the first-factor projection, let $X^{4}$ be the quotient of $K 3$ obtained by collapsing the fiber circles of $\pi_{1}:$

$$
X^{4}=K 3 / \sim,
$$


where

$$
\begin{aligned}
x \sim y \Leftrightarrow & x=y \text { or } \\
& x, y \in \pi^{-1}(p) \text { and } \pi_{1}(x)=\pi_{1}(y) .
\end{aligned}
$$

The space $X^{4}$ has a stratification $X_{4} \supset X_{1} \cong S^{1} \supset \varnothing$, and the link of $X_{1}$ is a 2 -torus, since the sphere-bundle of $T^{2} \hookrightarrow K 3$ is homeomorphic to $T^{3}$. Thus $X$ is not a Witt space. The quotient map $f: K 3 \rightarrow X$ is stratifiable, and is in fact an ordered resolution in the sense of Ban02, chapter 3. By Theorem 5.1 in Ban02, $S D(X) \neq \varnothing$, whence $X$ has a signature $\sigma(X)$. Now Theorem 6.3 computes that signature:

$$
\sigma(X)=\sigma(K 3)=16 .
$$

(The local system is the constant sheaf of rank 1 , since $f$ is a homeomorphism between top strata.)

\section{IMPLICATIONS FOR DESINGULARIZATION}

As we are working in the framework of Whitney stratifications, let us adopt the following definition: A closed, oriented, Whitney stratified pseudomanifold $X^{n}$ is resolvable by a stratified map if there exists a closed, oriented, smooth manifold $M^{n}$ which can be equipped with a Whitney stratification so that there exists a stratified map $f: M \rightarrow X$ whose restriction to the top stratum $f^{-1}(X-\Sigma)$ is an orientation-preserving diffeomorphism. The decomposition result, Theorem 6.1, can be used to identify obstructions to desingularizing a non-Witt space.

Theorem 7.1. If $X^{n}$ has a parity-separation, and there exists a field $\mathbb{k}$, char $\mathbb{k} \neq 2$, for which there is no Lagrangian structure (for constant coefficients) at the strata of odd codimension, then $X$ is not resolvable by a stratified map.

Proof. Suppose it is resolvable, and let $f: M \rightarrow X$ be a resolution. The constant sheaf $\mathbb{k}_{M}[n]$ is self-dual on $M$, since $M$ is oriented. The pushforward $\mathbf{S}^{\bullet}=R f_{*} \mathbb{k}_{M}[n]$ remains self-dual as $f$ is proper. By Theorem 6.1, $\mathbf{S}^{\bullet}$ is cobordant to a sheaf whose restriction to ${ }^{o} X$ is isomorphic to some $\left.\mathbf{I C}_{\mathcal{L}}{ }^{\circ} X ; \mathcal{S}\right)$, where $X={ }^{o} X \cup{ }^{e} X$ is a parity-separation. The coefficient system $\mathcal{S}$ on the top stratum of ${ }^{o} X$ is isomorphic to the constant sheaf with stalk $\mathbb{k}$, since $f$ is a diffeomorphism between top strata. Thus $\mathcal{L}$ is a Lagrangian structure for constant coefficients.

For example, let $X^{5}=\Sigma \mathbb{C} P^{2}$ be the suspension of complex projective space. The link of the two singular points of odd codimension is $\mathbb{C} P^{2}$, and there is no Lagrangian subspace in the middle cohomology $H^{2}\left(\mathbb{C} P^{2} ; \mathbb{k}\right)$. Thus $X^{5}$ is not resolvable by a stratified map. In this example, the obstruction is of a local nature, as the singular points are isolated. In general however, the obstruction to the existence of a Lagrangian structure is two-fold: The primary obstruction is the existence of a Lagrangian subspace at a base-point of the stratum, and the secondary obstruction is the existence of an invariant Lagrangian subspace with respect to the action of the fundamental group, which is a question of a more global nature. This secondary obstruction is not invariant under refinement of the stratification. For instance, one may have no Lagrangian structure around a circle stratum of odd codimension. Yet after insertion of a point into the circle, creating a new stratum of even codimension, the Lagrangian structure exists, because the circle minus the point is simply connected, so every locally constant sheaf over it is constant. 
Let us discuss an example (over $\mathbb{R}$ ) illustrating the secondary obstruction. An element $A \in S L_{2}(\mathbb{Z})$ defines a 3-manifold $M(A)$ fibered over a circle with fiber a torus and monodromy $A$. Let $p: M(A) \rightarrow S^{1}$ be the projection. The mapping cylinder $\operatorname{cyl}(p)$ is a 4-dimensional non-Witt pseudomanifold with boundary $M(A)$ and singular stratum $S^{1}$. There are Lagrangian subspaces in $H^{1}\left(T^{2}\right)$, and the primary obstruction vanishes. There is a $\pi_{1}\left(S^{1}\right)$-invariant Lagrangian subspace if and only if $A$ has a real eigenvalue. Let $A$ be an elliptic element such as

$$
A=\left(\begin{array}{cc}
1 & 1 \\
-1 & 0
\end{array}\right)
$$

with complex eigenvalues. To produce a closed object, choose an oriented 4manifold $W$ with $\partial W=M(A)$ and set

$$
X^{4}=W \cup_{M(A)} \operatorname{cyl}(p) .
$$

Then Theorem 7.1 asserts that $X^{4}$ is not resolvable by a stratified map.

Topological resolutions of singularities have been studied by M. Kato Kat73. and D. Sullivan Sul71, among others. Kato's theory can be briefly summarized as follows: If $P$ is a polyhedron, let $\Sigma$ denote the singular set. A PL $n$-variety is an $n$-polyhedron such that $P-\Sigma$ is a PL $n$-manifold without boundary. A PL blow-up between oriented PL $n$-varieties $\left(P^{\prime}, \Sigma^{\prime}\right)$ and $(P, \Sigma)$ is a proper PL map of pairs $f:\left(P^{\prime}, \Sigma^{\prime}\right) \rightarrow(P, \Sigma)$ such that for some derived neighborhoods $N^{\prime}$ of $f^{-1} \Sigma$ in $P^{\prime}$ and $N$ of $\Sigma$ in $P$, the restriction $f \mid: P^{\prime}-\operatorname{int}\left(N^{\prime}\right) \rightarrow P-\operatorname{int}(N)$ is an orientation-preserving homeomorphism. If $P^{\prime}$ is an oriented PL $n$-manifold, such a blow-up is called a PL resolution. This notion of resolution is weaker than resolution by a stratified map. Part of the definition of a stratified map requires that all of its restrictions to preimages of components of pure strata of the target are locally trivial fiber bundle projections. This structure is not required for Kato's PL resolutions. Now, given $(P, \Sigma)$, let $N$ be a regular neighborhood of $\Sigma$ in $P$. Its boundary $\partial N$ is an $(n-1)$-manifold. Let $p: \partial N \rightarrow \Sigma$ be the restriction of the normal projection $N \rightarrow \Sigma$. Then $p$ defines an oriented bordism class $[p] \in \Omega_{n-1}(\Sigma)$. Kato proves that $(P, \Sigma)$ admits a PL resolution if and only if $[p]=0$. The proof of the realization part is by attaching a nullbordism for $p$ to $P-\operatorname{int}(N)$ along $\partial N$. Since the extension of $p$ over the nullbordism is merely required to be a continuous PL map, the resulting resolution $f: P^{\prime} \rightarrow P$ is in general not a stratified map.

For $X^{5}=\Sigma \mathbb{C} P^{2},[p]=\left[\mathbb{C} P^{2} \rightarrow p t\right] \neq 0 \in \Omega_{4}(p t)$. Thus $X^{5}$ is not even PL resolvable. For the above $X^{4}$ we have to consider

$$
\left[M(A) \stackrel{p}{\longrightarrow} S^{1}\right] \in \Omega_{3}\left(S^{1}\right) .
$$

Since each $H_{n}\left(S^{1} ; \mathbb{Z}\right)$ is finitely generated and has no odd torsion, the bordism spectral sequence collapses and

$$
\Omega_{3}\left(S^{1}\right) \cong H_{0}\left(S^{1} ; \Omega_{3}(p t)\right) \oplus H_{1}\left(S^{1} ; \Omega_{2}(p t)\right)=0 .
$$

Consequently, $X^{4}$ admits a PL resolution, but not a resolution by a stratified map. Attempts to construct a stratified resolution by hand might include trying to "fill in" the torus by a smooth 3-manifold in a fiberwise fashion. As in [Kre84, let $\Delta_{*}$ denote the bordism groups of orientation-preserving diffeomorphisms of smooth closed manifolds. Matthias Kreck has proved that the bordism class of a diffeomorphism $f: M^{2 n} \rightarrow M^{2 n}$ is determined by three invariants: The bordism class of $M^{2 n}$, the bordism class of the mapping torus of $f$, and the isometric structure $I(M, f)=\left[H_{n}(M) /\right.$ Tor $\left., \phi, f_{*}\right]$ as an element in the Witt group $W_{(-1)^{n}}(\mathbb{Z} ; \mathbb{Z})$ of 
isometries of $(-1)^{n}$-symmetric unimodular bilinear forms on free finitely generated $\mathbb{Z}$-modules $(\phi$ is the intersection form on $M$ ). The space $M(A)$ is the mapping torus of the diffeomorphism $A: T^{2} \rightarrow T^{2}$ with isometric structure

$$
I\left(T^{2}, A\right)=\left[\mathbb{Z}^{2},\left(\begin{array}{cc}
0 & 1 \\
-1 & 0
\end{array}\right), A\right]
$$

This structure vanishes if and only if $|\operatorname{trace} A|=2$. Thus for nonparabolic $A$, $I\left(T^{2}, A\right) \neq 0$ and so $\left[T^{2}, f\right] \neq 0 \in \Delta_{2} \cong \mathbb{Z}^{\infty} \times \mathbb{Z}_{2}^{\infty}$.

\section{REFERENCES}

[Ban02] M. Banagl, Extending intersection homology type invariants to non-Witt spaces, Memoirs Amer. Math. Soc. 160 (2002), no. 760, 1 - 83. MR.1937924(2004e:55005)

[Ban05] - The L-class of non-Witt spaces, Annals of Math., to appear.

[BBD82] A. Beilinson, J. Bernstein, and P. Deligne, Faisceaux pervers, analyse et topologie sur les espaces singuliers, Astérisque 100 (1982), 1 - 171.

[BCS03] M. Banagl, S. E. Cappell, and J. L. Shaneson, Computing twisted signatures and Lclasses of stratified spaces, Math. Ann. 326 (2003), no. 3, 589 - 623. MR1992279 (2004i:32047)

[BK04] M. Banagl and R. Kulkarni, Self-dual sheaves on reductive Borel-Serre compactifications of Hilbert modular surfaces, Geom. Dedicata 105 (2004), 121 - 141. MR2057248 (2005d:32053)

[Che80] J. Cheeger, On the Hodge theory of Riemannian pseudomanifolds, Proc. Sympos. Pure Math. 36 (1980), 91-146. MR.0573430 (83a:58081)

[CS91] S. E. Cappell and J. L. Shaneson, Stratifiable maps and topological invariants, J. Amer. Math. Soc. 4 (1991), 521-551. MR1102578 (92d:57024)

[CSW91] S. E. Cappell, J. L. Shaneson, and S. Weinberger, Classes topologiques caractéristiques pour les actions de groupes sur les espaces singuliers, C. R. Acad. Sci. Paris Sér. I Math. 313 (1991), 293-295. MR.1126399 (92f:57035)

[GM83] M. Goresky and R. D. MacPherson, Intersection homology II, Invent. Math. 71 (1983), 77 - 129. MR0696691 (84i:57012)

[Kat73] M. Kato, Topological resolution of singularities, Topology 12 (1973), 355 - 372. MR 0339196 (49:3959)

[Kre84] M. Kreck, Bordism of diffeomorphisms and related topics, Lecture Notes in Math., no. 1069, Springer-Verlag, 1984. MR0755877 (86b:57015)

[Sul71] D. Sullivan, Singularities in spaces, Proceedings of the Liverpool Singularities Symposium-II, Lecture Notes in Math., no. 209, Springer-Verlag, New York, 1971, pp. 196 - 206. MR0339241 (49:4002)

Mathematisches Institut, Universität Heidelberg, Im Neuenheimer Feld 288, 69120 Heidelberg, Germany

E-mail address: banagl@mathi.uni-heidelberg.de 